\title{
Proposal of 3 Dimension Imaginary Space Touch Panel of Non-wearing Using Ultrasonic Sensors
}

\author{
Kohei Miyata $^{\mathrm{a},}$, Yuhki Kitazono $^{\mathrm{b}}$, Shiyuan Yang ${ }^{\mathrm{a}}$ \\ ${ }^{a}$ Kyushu Institute of Technology, 1-1, Sensui-cho, Tobata, Kitakyushu, Fukuoka, 804-8550 Japan \\ ${ }^{\mathrm{b}}$ Kitakyushu National Collage of Technology, 5-20-1 Shii, Kokuraminami-ku, Kitakyushu-city, Fukuoka 802-0985 \\ Japan
}

*Kohei Miyata: miyata@ boss.ecs.kyutech.ac.jp

\begin{abstract}
We propose three dimension virtual space touch panel that can be intuitively operated by moving the finger. In this research, three ultrasonic sensors are installed in the ceiling. Then, three dimension coordinates of the finger are specified by measuring the distance of each ultrasonic sensor from the finger. The simulation shows a good performance and accuracy. It is aimed to be used for $3 \mathrm{D}$ device operation.
\end{abstract}

Keywords: Sensing, Touch Panel, Ultrasonic Sensor.

\section{Introduction}

Three dimension display devices ${ }^{(1)}$ such as a head mounted display and a stereo television has been increased in recent years. Therefore, it is thought that a three dimension operating device ${ }^{(2,3)}$ is needed for sending an instruction to these products in the near future. Currently, three dimension operating devices that have been reported include a remote controller that treat the person's hand movement as an instruction ${ }^{(4,5)}$ and a three dimension space touch panel. Remote controller system for hand movement detection using cameras is complex, and easily causing a malfunction by a non-intended hand movement.

In addition, because the recognizable pattern of the hand movement is limited, only a few instructions can be assigned. Therefore, it is thought that the touch panel is more effective. There are various kinds of past touch panels. However, those shape is a plane, a curved surface, and the structure is basically a board. So they are two dimension devices in a real meaning. Such a structure cannot be extended to a three

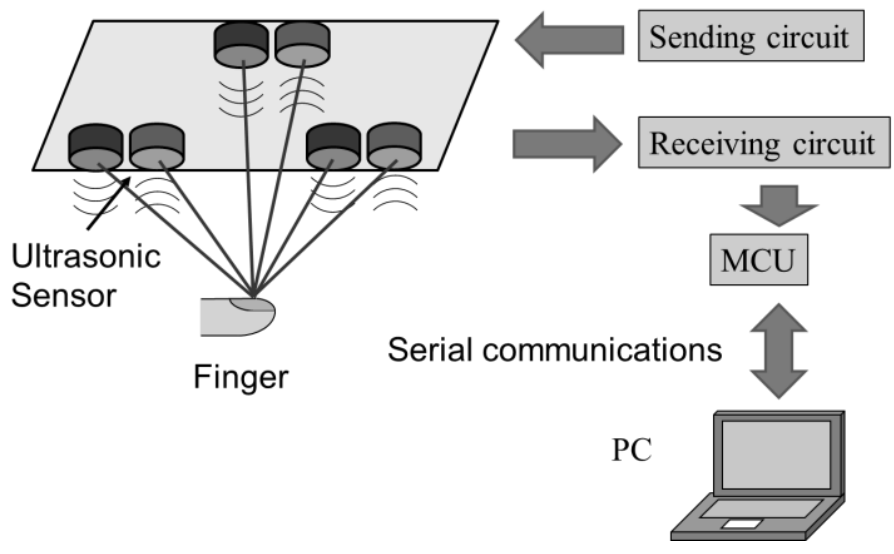

Fig. 1. System construction.

dimension device realistically, thus the one that use the three dimension space as the touch panel is not manufactured. Therefore, a three dimension visualization space touch panel using ultrasonic sensors is proposed in this research.

User can put the proposed virtual three dimension space touch panel at any convenient place where one condition that the ultrasonic wave is able to reach the ultrasonic sensors is cleared.

\section{System Details}

\subsection{System Configuration}

The system construction is shown in figure 1.First of all, the ultrasonic wave is sent from the ultrasonic sensor for the transmission installed in the ceiling, and reflected sound from finger is received by the ultrasonic sensor for the reception installed in the ceiling. The signals from the ultrasonic sensors for reception are taken into the 


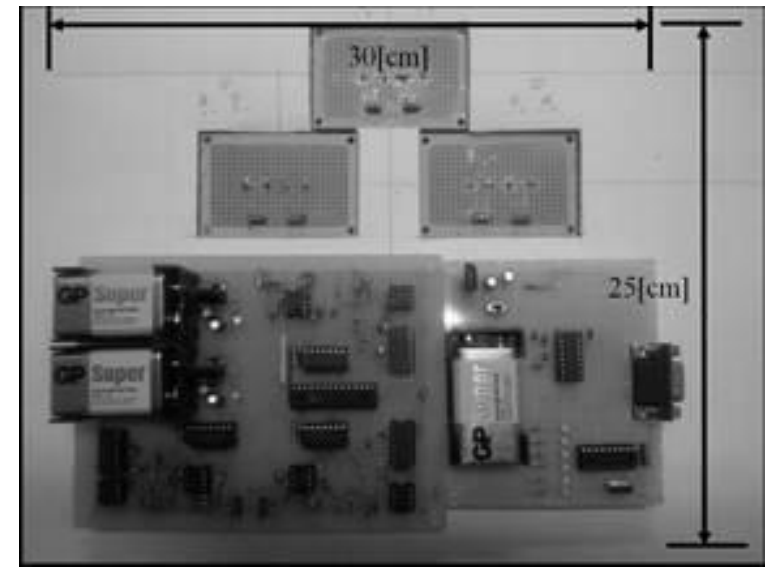

Fig. 2. Device.

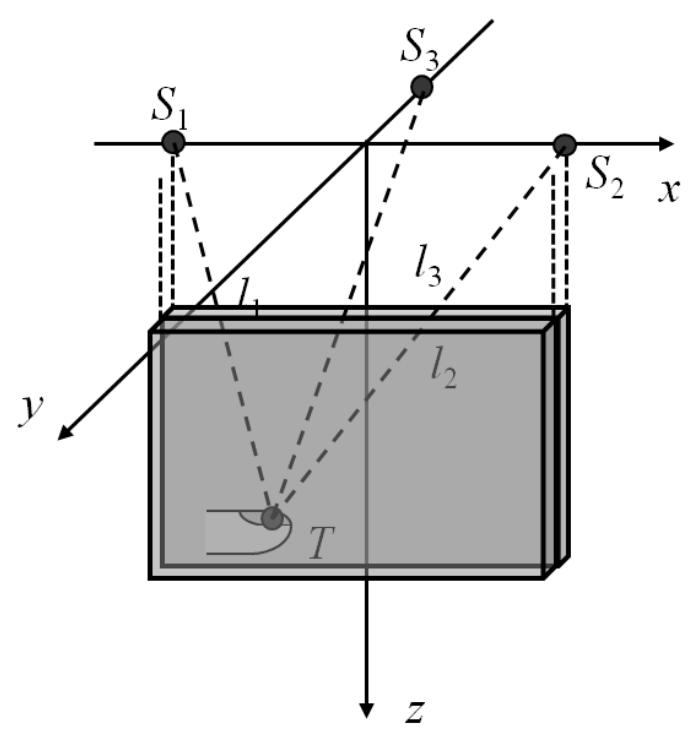

Fig. 3. Three dimension space touch panel.

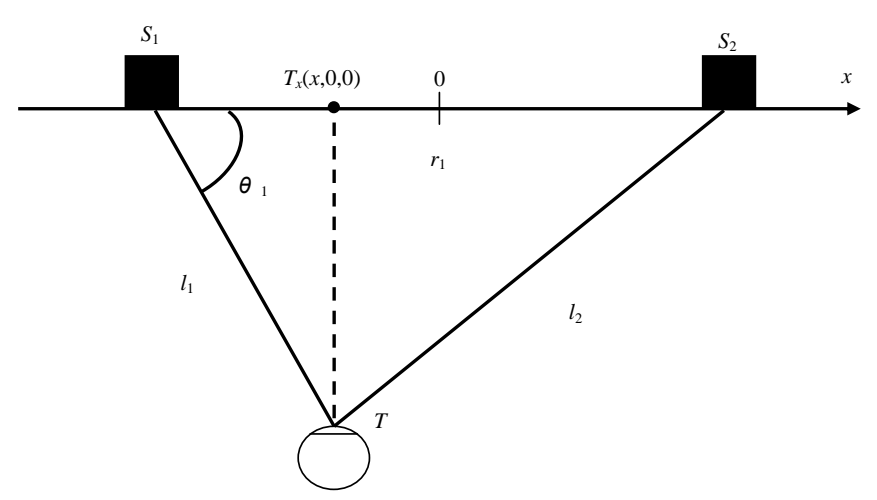

Fig. 4. $\quad x$ coordinates microcomputer, and the distance from the ultrasonic sensor to the finger is calculated. Then these distances are transmitted to the personal computer. The three dimension coordinates are worked out with the personal computer, and the pointer is displayed at where it should be in the 3-D space. Finally, whether the present pointer is on the position of a button or not is examined. Afterwards, if the pointer stops at the position of a button for two seconds or more, it is judged as a click event.

The ultrasonic sensor for transmission is shown in figure 2.Three ultrasonic sensors are used because it is necessary to calculate three dimension coordinates of the finger

The received signals are sent to the microcomputer and the distances (in this research is 3) are calculated soon once the signal was received.

\subsection{Deriving of Three Dimension Coordinates}

(a) Position of Ultrasonic Sensor for Reception

The position of ultrasonic sensor is shown in figure 3 . Assuming $S_{i}=\left(x_{i}, y_{i}, z_{i}\right)$.In this research, the ultrasonic sensors were set on three locations: $S_{1}=(-5,0,0), S_{2}=$ $(5,0,0)$, and $S_{3}=(0,-5,0)$. The unit is centimeter.

Where $l_{1}[\mathrm{~cm}]$ is the distance from finger $(T)$ to ultrasonic sensor at $S_{1}, l_{2}[\mathrm{~cm}]$ is the distance from finger $(T)$ to ultrasonic sensor at $S_{2}$, and $l_{3}[\mathrm{~cm}]$ is the distance from finger $(T)$ to ultrasonic sensor at $S_{3}$.

$$
l_{1}=\sqrt{(x+5)^{2}+y^{2}+z^{2}}
$$

$$
l_{2}=\sqrt{(x-5)^{2}+y^{2}+z^{2}}
$$

$$
l_{3}=\sqrt{x^{2}+(y+5)^{2}+z^{2}}
$$

\section{(b) Deriving of $\mathrm{X}$ Coordinates}

$x$ coordinates are calculated from the triangle that consists of $S_{1}, \quad S_{2}$ and $T$ of figure $4 . \quad x$ coordinates can be denoted by the expression (4).

$$
x=-5+l_{1} \times \frac{l_{1}^{2}+10^{2}-l_{2}^{2}}{2 \times 10 \times l_{1}}
$$




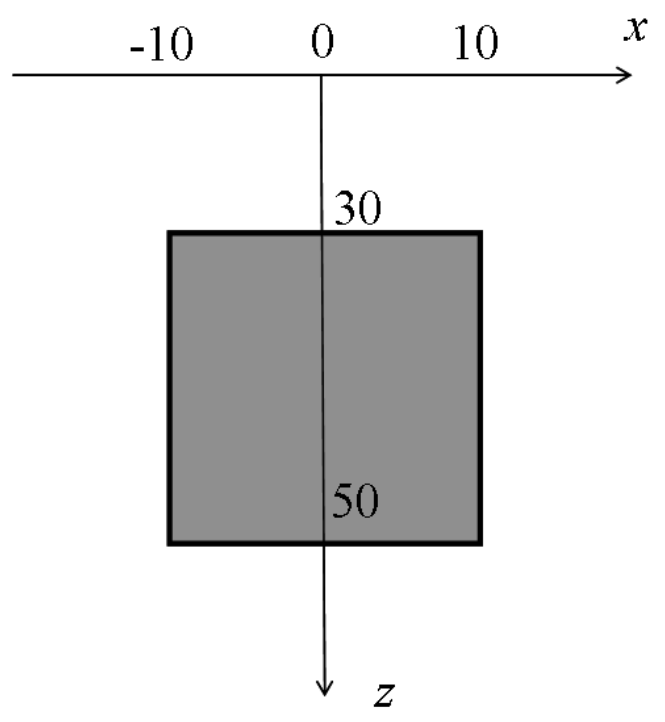

(a)Front view

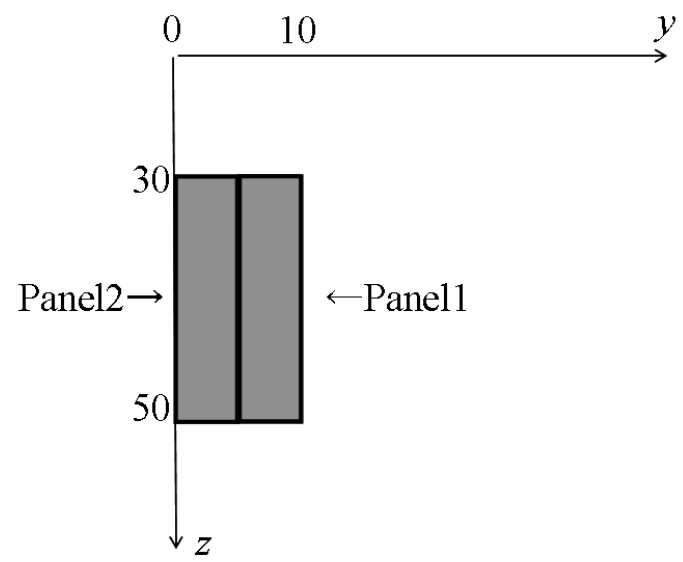

(b) Side view

Fig. 5. 3D imaginary space touch panel $(\mathrm{cm})$

Where $l_{1}[\mathrm{~cm}]$ is the distance from finger $(T)$ to ultrasonic sensor at $S_{1}, l_{2}[\mathrm{~cm}]$ is the distance from finger (T) to ultrasonic sensor at $S_{2}, l_{3}[\mathrm{~cm}]$ is the distance from finger ( $T$ ) to ultrasonic sensor at $S_{3}$, and $r_{1}[\mathrm{~cm}]$ is the distance from ultrasonic sensor at $S_{1}$ to ultrasonic sensor at $S_{2}$.

(c) Deriving of Y Coordinates

$y$ coordinates are calculated from figure 3 .

When $x$ is a positive number.

$$
y=-x-\frac{l_{2}^{2}-l_{3}^{2}}{10}
$$

When $x$ is a negative value.

$$
y=x-\frac{l_{1}^{2}-l_{3}^{2}}{10}
$$

Where $l_{1}[\mathrm{~cm}]$ is the distance from finger $(T)$ to ultrasonic sensor at $S_{1}, l_{2}[\mathrm{~cm}]$ is the distance from finger $(T)$ to ultrasonic sensor at $S_{2}$, and $l_{3}[\mathrm{~cm}]$ is the distance from finger $(T)$ to ultrasonic sensor at $S_{3}$.

(d) Deriving of $\mathrm{Z}$ Coordinates

$\mathrm{z}$ coordinates are calculated from figure 3 .

$$
z=\sqrt{l_{3}^{2}-x^{2}-(y+5)^{2}}
$$

Where $l_{3}[\mathrm{~cm}]$ is the distance from finger $(T)$ to ultrasonic sensor at $S_{3}$.

(e) 3 Dimension Imaginary Space Touch Panel

Traditionally, the surface of an existing board that shows buttons on it was used as a two dimensional touch panel. However, for three dimension space touch panel, the panel is not exist in real world, thus the touch panel needs to be defined virtual. Figure 5 shows the range of virtual touch panel which is called operating space. In this range, the ultrasonic sensors for reception can receive the ultrasonic wave generated by ultrasonic sensors for transmission well, so that the system works correctly.

\section{EXPERIMENT}

\subsection{Experimental Setup}

In this research, the distance is measured by the ultrasonic wave. However, the speed of sound changes according to the temperature changes. Therefore, it is necessary to correct the speed of sound according to the temperature changing. The correction is processed by the expression (8) and (9).

$$
v=33150+60 \times c
$$

$$
l_{t}=l v / 34000
$$




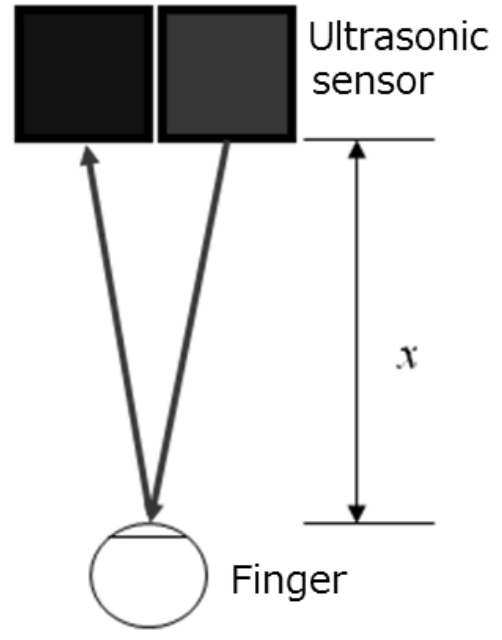

(a)Front view

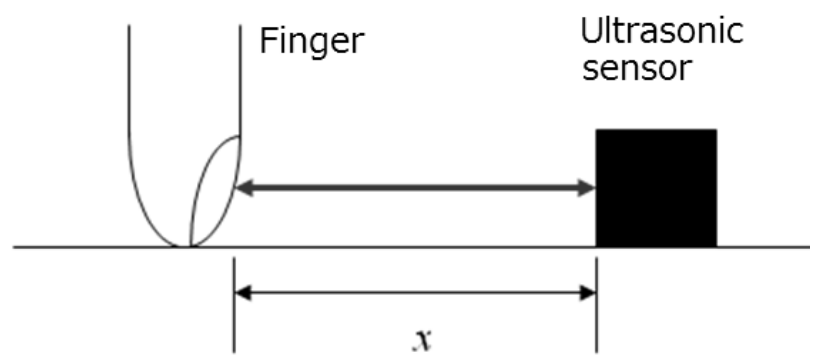

(b) Side view

Fig. 6. Experiment chart

Where $v[\mathrm{~cm} / \mathrm{s}]$ is the speed after the correction, $c$ is temperature $\left[{ }^{\circ} \mathrm{C}\right], l_{t}[\mathrm{~cm}]$ is distance after correction, and $l[\mathrm{~cm}]$ is distance before correction.

\subsection{Accuracy experiment of measurement distance}

Distance $\mathrm{x}$ is measured from 10 to $60[\mathrm{~cm}]$ at intervals of $5[\mathrm{~cm}]$, and theoretical values and measured values are compared as shown in Figure 6. The temperature when experimenting is $19{ }^{\circ} \mathrm{C}$. Figure 7 shows the experiment result.

\section{CONCLUSION}

In this research, a three dimension virtual space touch panel by using ultrasonic sensors was proposed and

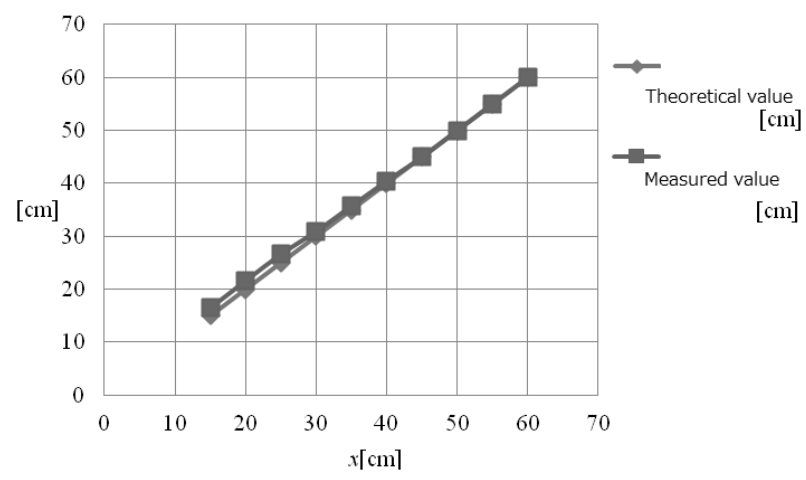

Fig. 7. Theoretical values and measured values

examined. It is effective though the idea is simple. The three dimension coordinates can be derived from three distances between ultrasonic sensors and finger very fast for practical use. Simulation result shows a good performance and accuracy.

In the future, we aimed to shrink the system scale for more widely application.

\section{References}

(1) G. Hamagishi, K.Taira, K.Izumi, S.Uehara, T. Nomura, K.Mashitani, A.Miyazawa, T.Koike, A.Yuuki, T.Horikoshi, Y.Yoshihara, Y.Hisatake, and H.Ujike, Y.Nakano : "Ergonomics for 3D Displays and Their Standardization”, IDW'08 Proc, pp.1099-1102, 2008.

(2) Takehiro Niikura, and Takashi Komuro : "3D touch panel interface using an autostereoscopic display", Proceedings of the 2012 ACM international conference on Interactive tabletops and surfaces, pp. 295-298, 2012

(3) Chih-Lung Lin, Chia-Sheng Li, Yi-Ming Chang, Chia-Che Hung, and Lin. A : "3D stylus and pressure sensing system for capacitive touch panel”, Journal of the 2012 IEEE International Conference on Consumer Electronics, pp. 215-216, 2012

(4) Fujii.R, and Komuro.T: "Zooming interface using a 3D finger position for mobile devices", Journal of the 2012 IEEE Symposium on 3D User Interfaces , pp. 143-144, 2012

(5) H. Hongo, M. Tominaga, Y. Niwa, and K. Yamamoto, "Control of household appliances in the Percept-Room”, ITE Technical Report, vol. 26, no. 44, pp.5-8, 2002. 